\title{
Venom evolution provides inspiration for development of ultrafast-acting insulins
}

$\underline{\text { John G. Menting }}^{1}$, Danny Hung-Chieh Chou ${ }^{2}$, Chris, A. MacRaild ${ }^{3}$, Nicholas, A. Smith ${ }^{4}$, Baldomero, M. Olivera ${ }^{5}$, Briony, E. Forbes ${ }^{6}$, Brian J. Smith ${ }^{4}$, Raymond S. Norton ${ }^{3}$, Helena Safavi-Hemami ${ }^{5}$, Michael C. Lawrence ${ }^{7}$

${ }^{1}$ Structural Biology Division, Walter And Eliza Hall Institute Of Medical Research, Parkville, Australia, ${ }^{2}$ Department of Biochemistry, University of Utah, Salt Lake City, United States, ${ }^{3}$ Monash Institute of Pharmaceutical Sciences, Parkville, Australia, ${ }^{4}$ La Trobe Institute for Molecular Science, La Trobe University, Bundoora, Australia, ${ }^{5}$ Department of Biology, University of Utah, Salt Lake City, United States, ${ }^{6}$ Department of Medical Biochemistry, Flinders University, Bedford Park, Australia, ${ }^{7}$ Walter and Eliza Hall Institute of Medical Research and Department of Medical Biology, University of Melbourne, Parkville, Australia E-mail: menting@wehi.edu.au

Investigation of peptide components in carnivorous cone snail venoms has given a boost to the development of new synthetic insulin therapeutics by the identification of fast-acting venom insulins distinct from endogenous snail insulin. One such insulin possesses a striking difference to other types present both in the snail and vertebrates in that it bears posttranslational modifications and, most striking, truncations that result in the absence of a B-chain C-terminal motif that has been shown to be essential for the engagement of insulin by the tandem-binding element that constitutes the primary binding site of the insulin receptor (IR). Whilst engagement of the cone snail insulin to human IR is substantially weaker than for native human insulin it nevertheless indicates that the cone snail insulin has features that somewhat overcome the absence of this motif [1]. For many decades therapeutic insulins have been developed to overcome the limitations of native insulin as a therapeutic agent. Here we build on the advances in our understanding of the engagement of insulin with its receptor that we made previously $[2,3]$ using X-ray crystallography to explore the means by which 50 million years of evolution have provided new avenues for insulin design. We provide insight into the structurally-directed design of ultrafastacting insulins fundamentally different from native and currently prescribed therapeutic insulins.

The figure below illustrates via molecular modelling how a cone snail insulin G1 (ConIns G1) might dock onto the IR L1 domain surface, the IR primary binding site. In black are shown human insulin residues from X-ray structure PDB 4OGA. In the absence of a homolog of the human insulin residue PheB24, rotation of the ConIns G1TyrB15 side chain may provide a surrogate, perhaps also requiring ConIns residue TyrB20.

(Image reproduced with permission from Menting et al. (2016) Nat. Struct. Mol. Biol. 23, 916-20)

[1] Menting J. G. et al. (2016). Nat Struct. Mol. Biol. 23, 916-920.

[2] Menting J. G. et al. (2014). Proc. Natl. Acad. Sci. U. S. A. 111, E3395-3404.

[3] Menting J. G. et al. (2013). Nature. 493, 241-24.

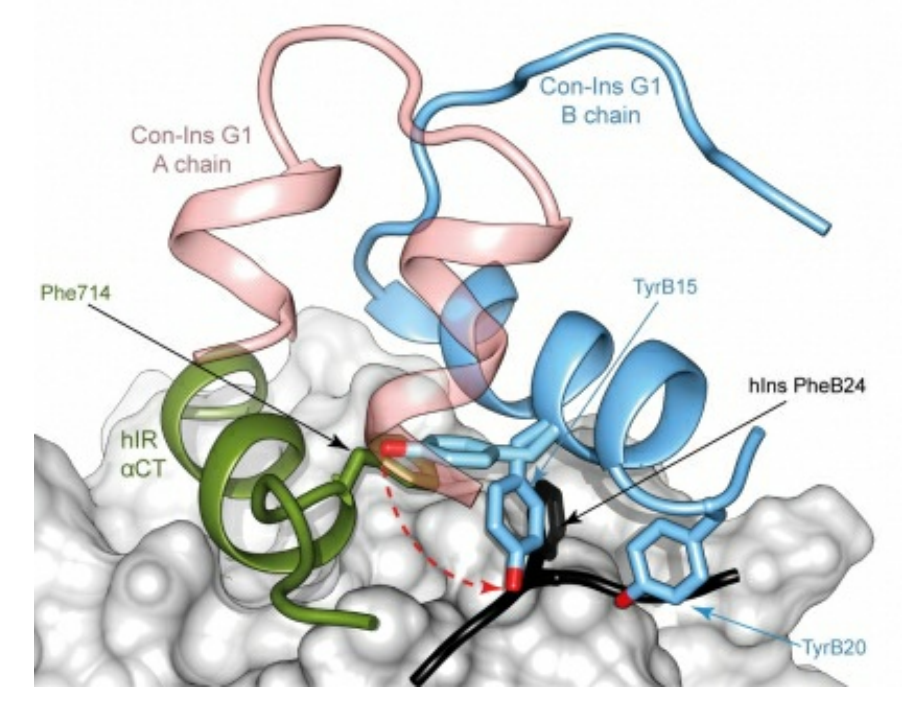

Keywords: diabetes mellitus, insulin receptor, cone snail venom insulin 